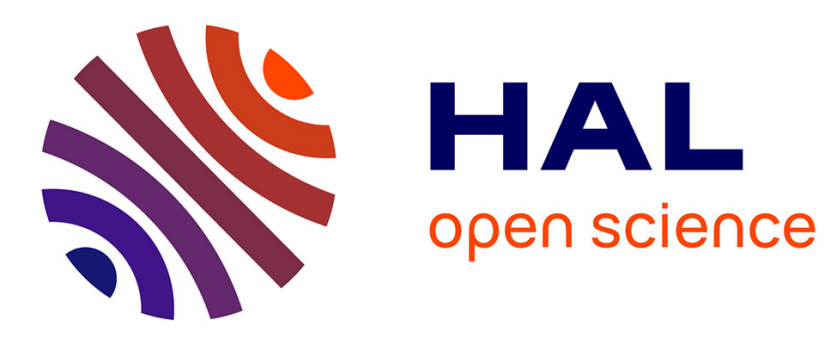

\title{
Bulletin du Commerce des bois
}

\author{
Emile Lachaussée, A. de Coincy, H. Mesnil
}

\section{To cite this version:}

Emile Lachaussée, A. de Coincy, H. Mesnil. Bulletin du Commerce des bois. Revue forestière française, 1956, 12, pp.875. 10.4267/2042/27266 . hal-03384032

\section{HAL Id: hal-03384032 \\ https://hal.science/hal-03384032}

Submitted on 18 Oct 2021

HAL is a multi-disciplinary open access archive for the deposit and dissemination of scientific research documents, whether they are published or not. The documents may come from teaching and research institutions in France or abroad, or from public or private research centers.
L'archive ouverte pluridisciplinaire HAL, est destinée au dépôt et à la diffusion de documents scientifiques de niveau recherche, publiés ou non, émanant des établissements d'enseignement et de recherche français ou étrangers, des laboratoires publics ou privés. 


\section{Bulletin du Commerce des Bois}

\section{Département du Jura}

Les ventes ont eu lieu:

- le 9 octobre à Dole, le II octobre à Lons-le-Saunier, le 15 octobre à Saint-Claude, le i7 octobre à Poligny.

Il a été vendu:

Bois fenillus:

I $865 \mathrm{~m}^{3}$ de bois d'œuvre et 65686 stères de bois de feu, soit des quantités à peu près égales à celles vendues en 1955 .

Bois résineux:

$94266 \mathrm{~m}^{3}$ de bois d'œuvre, $\mathrm{r} 946$ stères de bois d'industrie et 8234 stères de hois de feu. Les quantités de bois d'œuvre sont supérieures de $19 \%$ aux quantités vendues en 1955 .

12 articles seulement sur 776 sont restés invendus dans les forêts communales.

L'allure des cours est très différente suivant qu'il s'agit de feuillus ou de résineux.

Feuillus:

Bois d'œuvre: Les cours stationnaires pour le bois d'œuvre chêne, sont en très nette augmentation pour le bois d'œuvre hêtre $(+19 \%)$ et les très gros bois de feu (quartier, rondin $(+43 \%)$ à l'exclusion de la charbonnette dont la valeur reste toujours négative et est une charge pour l'exploitant.

La hausse du quartier rondin s'explique par les froids très vifs de février I956 qui ont 'permis la résorption des stocks et les difficultés momentanées pour les livraisons de charbon.

Résineux:

Les cours sont en très nette baisse par rapport à 1955 .

Pour le sapin, la baisse a été de:

- I0,5 \% à Lons-le-Saunier,

- $19,5 \%$ à Saint-Claude,

- I4,6 \% à Poligny,

soit en moyenne - I6 \%.

Cette baisse est due:

- aux trop hauts cours atteints en I955, qui étaient eux-mêmes en hausse de 6i \% par rapport aux cours de 1954, alors que les prix de ventes des sciages qualité charpente ont baissé, mettant les scieurs dans des situations critiques.

- une augmentation du volume mis en vente: $+14866 \mathrm{~m}^{3}$, soit ig \% en plus par rapport à 1955 .

Les ventes ont été l'objet d'une faible concurrence à Dôle, Lons-le-Saunier et Saint-Claude, mais la compétition a été extrêmement vive à Poligny, tant en raison de l'importance de la vente (393 articles) que de sa date, car c'était la dernière grande vente en Franche-Comté.

E. Lachaussée. 


\section{Département de la Gironde}

L'adjudication du 22 octobre 1956 comprenait:

40 articles cubant au total $6 \mathrm{I} 185 \mathrm{~m}^{3}$, contre $5 \mathrm{I} 633 \mathrm{~m}^{3}$ en $\mathrm{I} 955$.

2 articles d'un volume total de $509 \mathrm{~m}^{3}$ sont restés invendus (petits bois d'industrie).

II n'a pas été mis en vente de coupes à sciage de $\mathrm{I}^{\text {re }}$ catégorie.

Les coupes à isciage de $2^{\theta}$ catégorie se sont vendues en moyenne $277 \mathrm{I} \mathrm{F}$ le $\mathrm{m}^{3}$ (tous frais compris) - forêts domaniales: $2687 \mathrm{~F}$, forêts communales : $3046 \mathrm{~F}$. La hausse moyenne sur I955 est de I3 \%.

Les coupes de régénération des séries de protection formées de bois médiocres ont trouvé preneur à $2089 \mathrm{~F}$ le $\mathrm{m}^{3}$.

Le prix moyen des bois d'industrie a été de $2199 \mathrm{~F}$ (+ I4 \%). Dans ce chiffre entrent les coupes de très petits bois, destinés à la papeterie. A I $216 \mathrm{~F}$ le $\mathrm{m}^{3}$, leur hausse atteint $34,5 \%$. Ce prix est à souligner, car il facilitera aux propriétaires l'exécution des travaux culturaux dans les jeunes pins.

Les bois d'industrie de plus fortes dimensions ont été payés $2477 \mathrm{~F}$ en coupes de régénération domaniales; i $843 \mathrm{~F}$ et I $958 \mathrm{~F}$ dans les coupes d'éclaircies domaniales et communales (hausse ro \% environ).

Le produit total de la vente frais compris, a été de $150200000 \mathrm{~F}$. Elle ne comprenait aucune coupe de qualité exceptionnelle.

A. de Coincy.

\section{Département des Landes}

Les ventes des Inspections de Dax et Mont-de-Marsan ont eu lieu les 20 et $3 a$ octobre 1956 .

Elles portaient sur $4639 \mathrm{~m}^{3}$ de feuillus (chêne principalement) et III $020 \mathrm{~m}^{3}$ de pin maritime.

Feuillus:

A Dax, les chênes se sont vendus en moyenne $3967 \mathrm{~F}$, soit sensiblement le même prix qu'en 1955 avec maximum de $7000 \mathrm{~F}$.

A Mont-de-Marsan, le prix moyen a été de $3677 \mathrm{~F} \mathrm{le} \mathrm{m}^{3}$.

Le bois de chauffage a atteint I2o $\mathrm{F}$ le stère.

Pin maritime:

Les coupes de régénération (sciage) de $\mathrm{I}^{\text {re }}$ catégorie de l'Inspection de Dax ont 'été payées $3504 \mathrm{~F}$ le $\mathrm{m}^{3}$ (hausse de ro \% sur 1955). A Mont-deMarsan, le prix correspondant a été de 3 I $54 \mathrm{~F}$ (forêts domaniales) et $3670 \mathrm{~F}$ (forêts communales) $(+20 \%)$. Le prix le plus élevé a été 4 Ioo $\mathrm{F}$ le $\mathrm{m}^{3}$ à Mimizan.

Les coupes de sciage de $2^{\mathrm{e}}$ catégorie ont donné à $\operatorname{Dax} 2643 \mathrm{~F}(+30 \%)$ et à Mont-de-Marsan $2742 \mathrm{~F}(+8 \%)$ en forêts domaniales et $3204 \mathrm{~F}$ en forêts communales.

Pour les bois d'industrie de 0,60 à 0,90 , le prix à Dax a été de I $883 \mathrm{~F}$ $(+7 \%)$ et celui de Mont-de-Marsan $2020 \mathrm{~F}$ en forêts domaniales (+ $15 \%$ ). Les coupes rases de bois d'industrie ont atteint $2586 \mathrm{~F}$ à Mont-de-Marsan $(+57 \%)$.

Les petits bois d'industrie qui vont surtout à la papeterie ont accusé, à Dax, une hausse de $32 \%$ (prix: I $577 \mathrm{~F} \mathrm{le} \mathrm{m}^{3}$ ) tandis qu'à Mont-de-Marsan ces éclaircies de très jeunes bois ont donné I $464 \mathrm{~F}$ (hausse: $2 \%$ ).

Très peu d'articles sont restés invendus (4 sur I63).

En résumé, les ventes des Landes ont confirmé une tendance à la hausse, par rapport à 1955, tendance qui s'était déjà manifestée depuis le printemps dernier, au cours des ventes communales ou particulières.

Elle est particulièrement marquée sur les bois d'industrie.

A. de Corncy. 


\section{Place de Compiègne}

La vente des coupes de bois de l'exercice 1956 de l'Inspection de Compiègne a eu lieu le mardi 30 octobre, à Compiègne.

Cette adjudication figurait en fin de calendrier des grandes ventes annuelles, non seulement de la $3^{\circ}$ Conservation, mais aussi des conservations voisines.

On s'attendait à une vive compétition, de nombreux professionnels du bois, étrangers à la région, ayant visité les coupes, ou tout au moins acheté le cahier-affiche.

De fait, l'assistance était nombreuse et attentive.

Grâce à l'autorité et au remarquable doigté du Sous-Préfet de Compiègne, Président de séance, celle-ci s'est déroulée dans une excellente ambiance et sans le moindre incident.

Il est, d'autre part, certain que les conseils de prudence et de modération prodigués, la veille de l'adjudication, par le Président de l'Union Syndicale des Marchands de Bois et Exploitants Forestiers, ont porté leurs fruits .

L'affiche était très bien fournie.

Elle comprenait I74 articles, à savoir :

\section{Forêts domaniales:}

I4I coupes de futaie pouvant produire ensemble 57933 mètres cubes et 34.753 stères ;

4 coupes de taillis-sous-futaie, d'une contenance de' 56 ha 05 a, pouvant produire ensemble I 433 mètres cubes et ro 040 stères;

22 coupes de produits accidentels pouvant produire ensemble I 777 mètres cubes et 916 stères.

Forêts communales:

5 coupes de taillis-sous-futaie, d'une contenance d I5 ha 24 a pouvant produire ensemble 618 mètres cubes et $\mathrm{I} 260$ stères;

2 coupes de futaie affouagère pouvant produire ensemble II 6 mètres cubes et 85 stères.

Dans l'ensemble, les produits offerts étaient de qualité homogène, avec quelques très beaux lots de hêtre situés sur les monts ceinturant la Forêt de Compiègne, et de beaux spécimens de chêne issus de plantations, dans les parties basses de la même forêt.

Les éclaircies, pourvues surtout en bois de faibles dimensions. renfermaient des produits de bonne qualité.

Les lots des Forêts domaniales de Laigue, Ourscamp-Carlepont, et l'Hòpital, contenaient. cette année, quelques beaux spécimens de chêne à grain tendre. et aussi des hêtres et des divers de belle venue.

Six lots seulement n'ont pas trouvé preneur, dont un de produits accidentels.

En ce qui concerne les prix unitaires, les cours des bois de chêne sont très fermes; les qualités tranchage sont toujours recherchées mais leur prix est stable, alors que les bois de qualité ébénisterie, menuiserie, charpente et parquets, s'enlèvent à des prix supérieurs de $15 \%$ à ceux de l'an dernier.

Les cours du hêtre sont aussi très fermes. Les belles billes de hêtre sont dirigées vers le déroulage et se négocient facilement autour de 8000 franıs le mètre cube, prix atteint déjà l'an dernier.

Les bois de moyenne et faible dimension marquent une hausse d'environ I5 \%.

Les bois d'œuvre divers ont trouvé facilement preneur à environ I 000 francs le mètre cube, prix également en hausse d'environ $20 \%$.

L'affiche ne comprenait que fort peu de résineux, dont les cours accusent une augmentation de $10 \%$. 
Les cours des bois de mine d'essence dure sont restés stables.

Les bois de papeterie s'estiment autour de 200 francs le stère.

Quant au bois de chauffage, il connait un certain renouveau d'activité: quartier et rondin se sont estimés sur la base de 100 francs le stère.

Les prix atteints ont été très voisins des estimations: les I4I lots adjugés des forêts domaniales l'ont été pour un prix global de I8I 000000 , alors que les estimations se montaient à 177000000 .

Les prix du mètre cube sur pied, charges comprises et taxes non comprises, se résument comme suit:

Chêne:

Tranchage (ordinaire) $\ldots \ldots \ldots \ldots \ldots \ldots, 30000$

$\mathrm{I}^{\mathrm{r}}$ qualité (ébénisterie) $\ldots \ldots \ldots \ldots \ldots \ldots \ldots$ I4 000

$2^{\natural}$ qualité (menuiserie et charpente) $\ldots \ldots \ldots, 6500$

$3^{\circ}$ qualité (traverses et parquets) $\ldots \ldots \ldots \ldots$ I 800

Hêtre:

Déroulage

$\mathrm{I}^{\text {re }}$ qualité (beà sciage)

$2^{\mathrm{e}}$ qualité (sciage ordinaire) $\ldots \ldots \ldots \ldots \ldots \ldots \ldots \ldots \ldots \ldots \ldots \ldots$

$3^{\circ}$ qualité (traverses) $\ldots \ldots \ldots \ldots \ldots \ldots \ldots$ I 200

Divers:

Bois d'œuvre (sciage ordinaire)

Résineux:

Bois d'œuvre (beau sciage)

Bois de mine:

Le stère

Bois de papeterie:

Le stère

Bois de feu:

Le stère
Pourcentage moyen de hausse ou de baisse

Hausse Baisse

Sans changement

I5 \%

I5 \%

I5\%

Sans changement $15 \%$

$15 \%$

$20 \%$

I $000 \quad 20 \%$

$4400 \quad$ 10 $\%$

600 Sans changement

$200 \quad 100 \%$

I00 Non estimé en I955

En résumé:

- Hausse marquée sur les bois de chêne; stabilité, cependant du cours du tranchage;

- Hausse marquée des cours du hêtre; stabilité du déroulage;

- Hausse des bois d'œuvre d'essences diverses et résineuses;

- Cours très fermes des hois de papeterie;

- Revalorisation des bois de feu.

H. MEsNiL.

Les anciennes collections de la Revue Forestière Française sont recherchées par de nombreux amateurs.

Les abonnés qui veulent s'en dessaisir sont invités à nous le faire savoir. 